\title{
Search for Keplerian periods in light variations of T Tauri stars and Herbig Ae stars
}

\author{
S. A. Artemenko, K. N. Grankin, P. P. Petrov \\ Crimean Astrophysical Observatory, Ukraine
}

Long-term, uniform series of UBVR observations of T Tauri and Herbig Ae stars obtained over $20 \mathrm{yr}$ at the Maidanak Observatory as part of the ROTOR program are analyzed. We find a linear relationship between the characteristic variability time scale and the bolometric luminosity of the star+disk system: the higher the luminosity, the slower the brightness variations. This dependence is valid over a wide range of masses and luminosities, from $\mathrm{T}$ Tauri stars to Herbig Ae stars. On average, the variability time scale is one-quarter the Keplerian period at the dust-sublimation radius, which is known from interferometric observations. Some T Tauri stars have periods from 25 to 120 days, which are preserved over several observing seasons. These periods correspond to Keplerian orbits with semi-major axes from 0.14 to 0.52 AU. The results obtained provide indirect evidence for the existence of protoplanets in the gas-dust disks of stars in early stages of their evolution toward the main sequence.

\section{Introduction}

The young pre-main sequence objects are known as irregular variables. By their physical parameters they fall into two large groups: the $\mathrm{T}$ Tauri stars with masses $\leq 2.5 \mathrm{M}_{\odot}$, and the Herbig Ae-Be stars with masses $1.5-15 \mathrm{M}_{\odot}$. These groups differ not only in mass and luminosity, but also in internal structure of stars: unlike Herbig Ae-Be stars, T Tauri stars have outer convective envelopes. Detailed description of the observed characteristics of T Tauri stars and Herbig Ae-Be stars can be found in review papers [1-3]. The young stars possess circumstellar gaseous dusty dusks, where the process of planetary system formation is presumably going on. In recent years, the protostellar disks have been investigated intensively by means of optical interferometry [4]. In this paper we consider photometric variability of the Herbig Ae stars (HAeS) and the classical T Tauri stars (cTTS) with accretion disks.

The two main sources of the irregular light variability of young stars are 1) extinction and scattering of light in dust clouds of circumstellar disk, and 2) accretion processes: infall of matter on stellar surface is accompanied by energy liberation, which changes the apparent stellar brightness. The accretion processes are more evident in cTTS of late spectral types, where the accretion luminosity is comparable to stellar luminosity and sometimes even exceeds it. On the other hand, the circumstellar extinction is more noticeable in HAeS with more massive dusty circumstellar disks. Many HAeS shows irregular deep minima of brightness, caused by obscuration of star by dust clouds. When a star is sufficiently screened out by dust, contribution of scattered light increases which results in rise of linear polarisation and sometimes in bluer colours (UX Orionis phenomenon, [5]). The photospheric spectrum of such star remain unchanged. Some of cTTS of spectral types earlier than K2 also show the effect of UX Ori. The role of different mechanisms of variability of cTTS was discussed in the recent paper by Grankin et al.[6]. One more reason of photometric variability is the magnetic activity, which reveal itself, specifically, in appearance of cool spots on stellar surface. This effect is present only in stars with convective envelopes. Due to long life time of the cool spots, they 
can be discovered from steady rotational modulation of brightness with periods of several days $[7]$.

A protoplanetary disk is not uniform. The presence of graviting bodies in the disk result in regular perturbations of density, which can be detected from periodical brightness variations, provided a favorable orientation of the disk to the line of sight. The density waves can appear not only on the disk plane, but also in the disk wind [8], which increases the probability of their detection. Herbig Ae stars seem to be the most promising objects to look for such periodicities. Light curves of the stars were analysed in details in [9-15], but no stable periods were detected. At longer time intervals of months and years, wave-like variations of the maximum level of brightness were found. In some stars a cyclic pattern of these variations was revealed (e.g., in SV Cep [12]). Such photometric behavior indicates existence of stable structures in the circumstellar disk, caused by presence of either low-mass secondary or a protoplanet $[8,15]$.

The most uniform time-series of cTTS and HAeS were obtained in course of the ROTOR project [16] in 1983-2004 at the Majdanak Observatory, Uzbekistan. The time series of 10-20 years provide a rich database to study variability on time scales of 20 to 200 days, corresponding to Keplerian periods near the inner radius of the accretion disks. In this paper we report on search for such periodicities in light variations of cTTS and HAeS.

\section{Observational data and methods of analysis}

The observational data were described by Grankin et al. [6] and are available in electronic form from CDS, Strasbourg (anonymous ftp to sdsarc.u-trasbg.fr). The observations of HAeS before 1999 were published in [17]; later observations are not published yet. All the data were reduced to the Johnson's UBVR system. For stars brighter than V=12 mag the standard deviation is $0.01 \mathrm{mag}$ in BVR and $0.05 \mathrm{mag}$ in U. From the list of targets in the catalogue we selected those objects which were observed during at least five seasons (maximum 23 seasons), with duration of one season from 3 to 6 months. Usually, each target was observed once in every clear night during a season. Typical light-curves of HAes and cTTS are shown in Fig.1.

In this paper we analyse $11 \mathrm{HAeS}$ showing effect of UX Ori, and 28 cTTS. All the light-curves of these stars show both the short term nigh-to-night variations and the long term variations from season to season. We are interested in probable periods on time scale from 20 to 200 days, therefore variations on longer time scales are considered as a low-frequency trend. In order to remove the trend we subtracted the seasonal average from the data within each season. The light variations may be caused by both the periodical and stochastic processes, therefore we used two different methods: the digital spectral analysis and the method of auto-correlation functions.

\section{Digital spectral analysis}

In order to reveal the hidden periodic processes from the light curves we used different methods of the power spectra estimations: CLEAN [18], Chi-square [19], SCARGLE [20] and the classical correlogram method CORRPSD [21]. The statistical characteristics of the power spectra calculated by these methods appeared about the same, not counting small differences in the form, therefore in the following we show and discuss only the results from the CORRPSD method. 

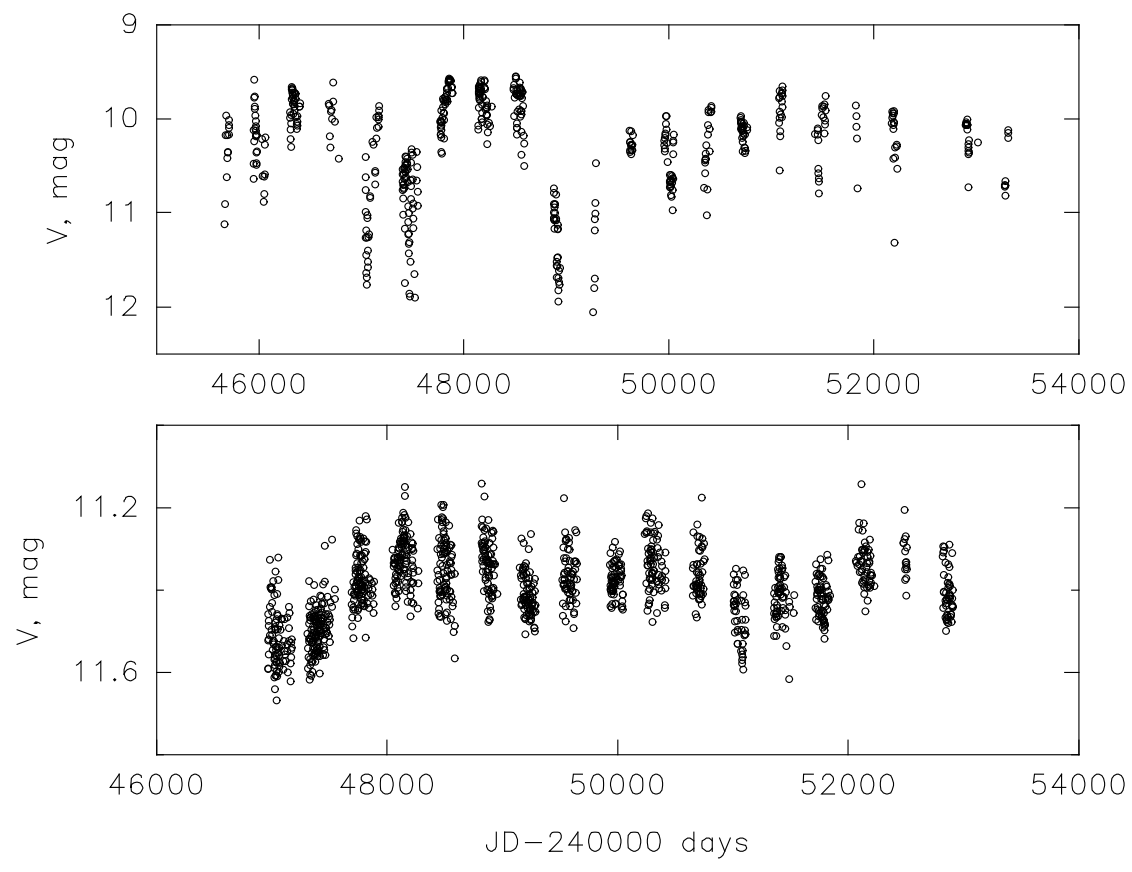

Figure 1: Typical light-curves of HAes and cTTS on time interval of about 20 years. Upper panel: BF Ori, spectral type A2. Lower panel: DI Cep, spectral type G8.

From the point of view of the spectral analysis theory, our light curves represent discrete, unevenly sampled and rather short time sequences of data with significant seasonal gaps. These properties result in badly predicted distortions of the corresponding estimates of the power spectra and makes it impossible to reconstruct the true (consistent) power spectra. In other words, the estimate of a power spectrum appeared to be unstable or inconsistent. For reconstruction of consistent estimate we used the method of smoothing the spectral estimate, as was first suggested in [22] for the case of correlogram method. Detailed discussion of the problems of reconstruction of consistent power spectrum in case of real astronomical time-series can be found in [23].

We used the smoothing algorithm described in [24]. The smoothing of power spectrum was done by change in the correlation window width. Narrowing the correlation window leads to smaller variance of spectral estimate at the expense of spectral resolution. The problem is to find a compromise between the spectral resolution and the consistency of spectral estimate. We use the classical CORRPSD method and the Tukey window function. In this method, in computation of a power spectrum one uses not the values of time series but a finite sequence of auto-correlation function.

The Fig.2 shows typical power spectra of HAeS and TTS. Unlike HAeS, power spectra of many TTS contain peaks corresponding to the periods of axial rotation ( $<10$ days). The rotational modulation of light of cTTS is due to the cold and hot spots on their surface. We reserve the analysis of the rotational periods and physical characteristics of the spots for another paper. Here we consider processes of lower frequencies, with characteristic time $>10$ days. It is known that light variations of HAeS is usually slower than that of TTS [25]. This can be noticed from the shape of the power spectra in Fig.2: in HAeS the power is rising steeply towards the 

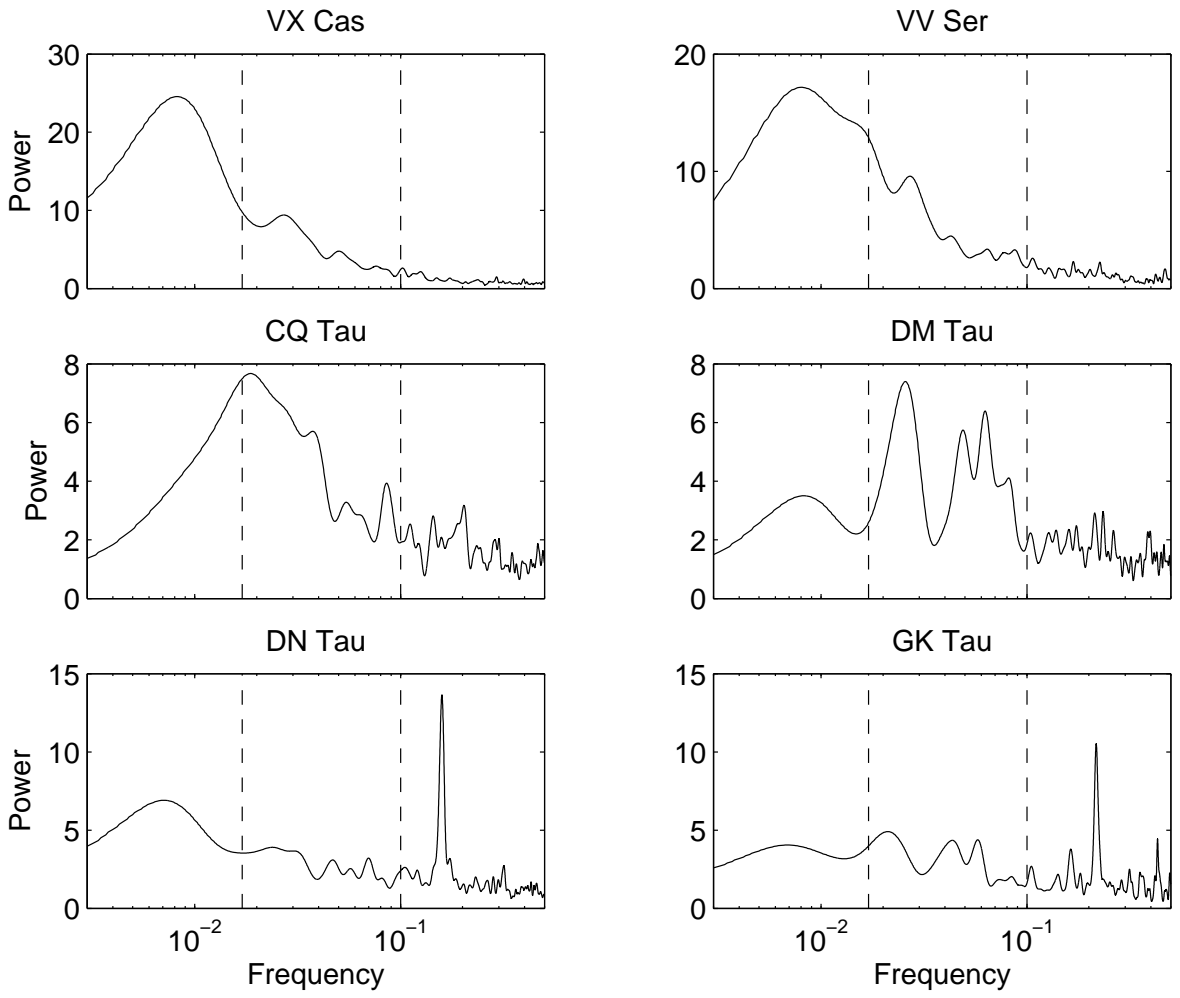

Figure 2: Examples of power spectra of HAeS (VX Cas and VV Ser) and cTTS (CQ Tau, DM Tau, DN Tau and GK Tau). The dashed lines mark the frequency intervals used for determination of the colour index of the power spectra.

lower frequencies, while in TTS the spectrum is more flat. Let's express this quantitatively. By analogy with the energy distribution in optical spectrum of a star, one can apply the method of colorimetry to the power spectrum. The summed power within selected frequency bands can be measured, and the colour indexes can be determined. We selected two bands: the low-frequency band from 0.005 to 0.0166 1/day (periods from 60 to 200 days), and the mid-frequency band from 0.0166 to 0.01 (periods from 60 to 200 days). The boundaries of these two bands are marked by dashed lines in Fig.2 Let's denote the summed power within the low-frequency band as LF, and the summed power within the mid-frequency band as MF. Then, the ratio LF/MF is analog of the red colour index: the larger $\mathrm{LF} / \mathrm{MF}$, the longer the characteristic time of variability. In case there is a periodical component in light variations, the LF/MF ratio depends on the value of the period.

\section{Auto-correlation functions}

Variability of a star can be characterised also by means of auto-correlation function (ACF). When calculating the ACF, we remove the seasonal trend by the same way as in calculation of power spectrum. Besides of that, in those cases when a period of axial rotation is clearly present in the brightness variations, the periodical component was also removed from the data set before calculating the ACF. We calculated the ACF from all the light-curves in B, V and 


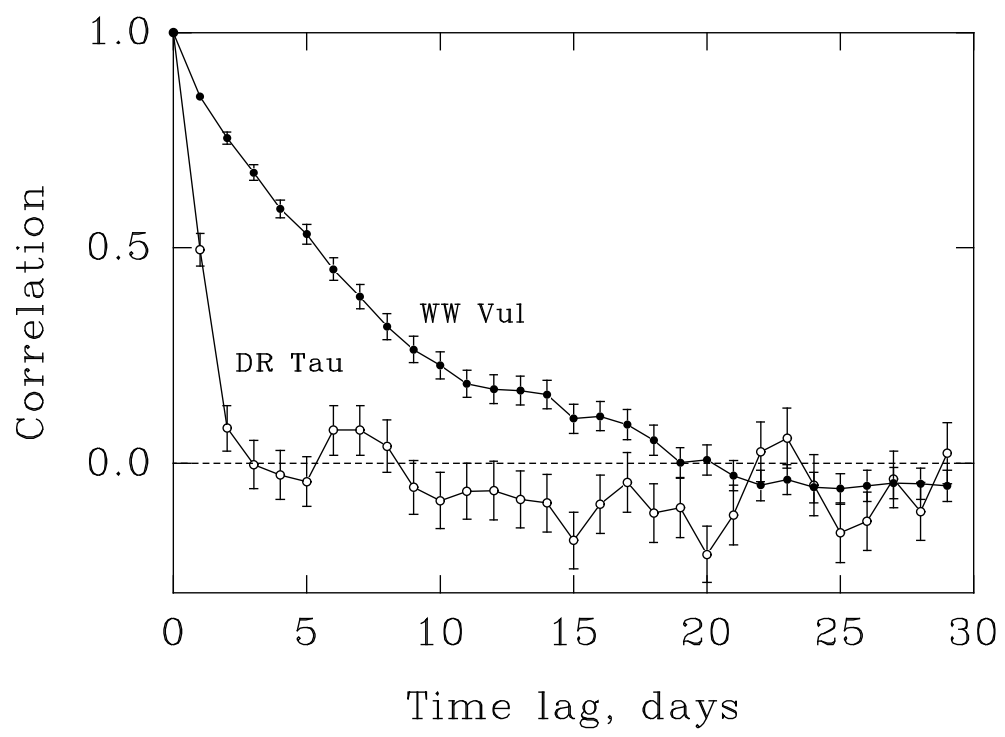

Figure 3: Auto-correlation functions of light curves of HAeS WW Vul and cTTS DG Tau

$\mathrm{R}$ bands. All the three ACFs are about the same, so in the following we discuss only the ACF calculated for the V band. As an example, ACFs for DR Tau (cTTS) and WW Vul (HAeS) at the time interval of 30 days are shown in Fig. 3. Apparently, the widths of the central peak of these ACFs are different, which indicates the different rates of brightness variations.

Lets denote the width of ACF at zero level as W0. This parameter, sometimes called "interval of correlation", is the time interval during which stellar brightness changes from mean level to maximum or minimum. In case of sinusoidal variations with period $\mathrm{P}$ this interval equals to $1 / 4 \mathrm{P}$. In case of non-periodical variations, $\mathrm{W} 0$ can be considered as a characteristic time of variability. In the following we will use the term "characteristic time" in this meaning.

\section{Relationship between the characteristic time and luminosity}

The measured values of $\mathrm{LF} / \mathrm{MF}$ and $\mathrm{W} 0$ are given in Table 1, together with errors in W0. The errors in LF/MF is typically less than $1 \%$. Both parameters characterise the same property: the mean rate of light variability, or the characteristic time of light variability. The average value of W0 is 8.7 days for the group of cTTS, and 21.1 days for the group of HAeS.

When considering global stellar parameters, like mass and luminosity, we discovered dependence of both LF/MF and W0 from the total bolometric luminosity of the system "star + disk" (Fig.4). This luminosity, denoted as $\mathrm{L}_{t o t}$, includes the bolometric luminosity of the star and the luminosity caused by accretion, from optical to IR down to $100 \mathrm{mkm}$. the total bolometric luminosities and the corresponding references $[26,27,29,35,36]$ are given in columns 2 and 3 in Table 1. The Fig. 4 shows that both cTTS and HAeS groups comprise a common dependence: the larger the luminosity is, the slower the variability. The range of 2-3 dex in luminosity corresponds to the range of 1 dex in the characteristic time of variability. Probably, the characteristic time of variability is related to the disk size.

For the last decade, large progress was achieved in measurements of the inner radii of 

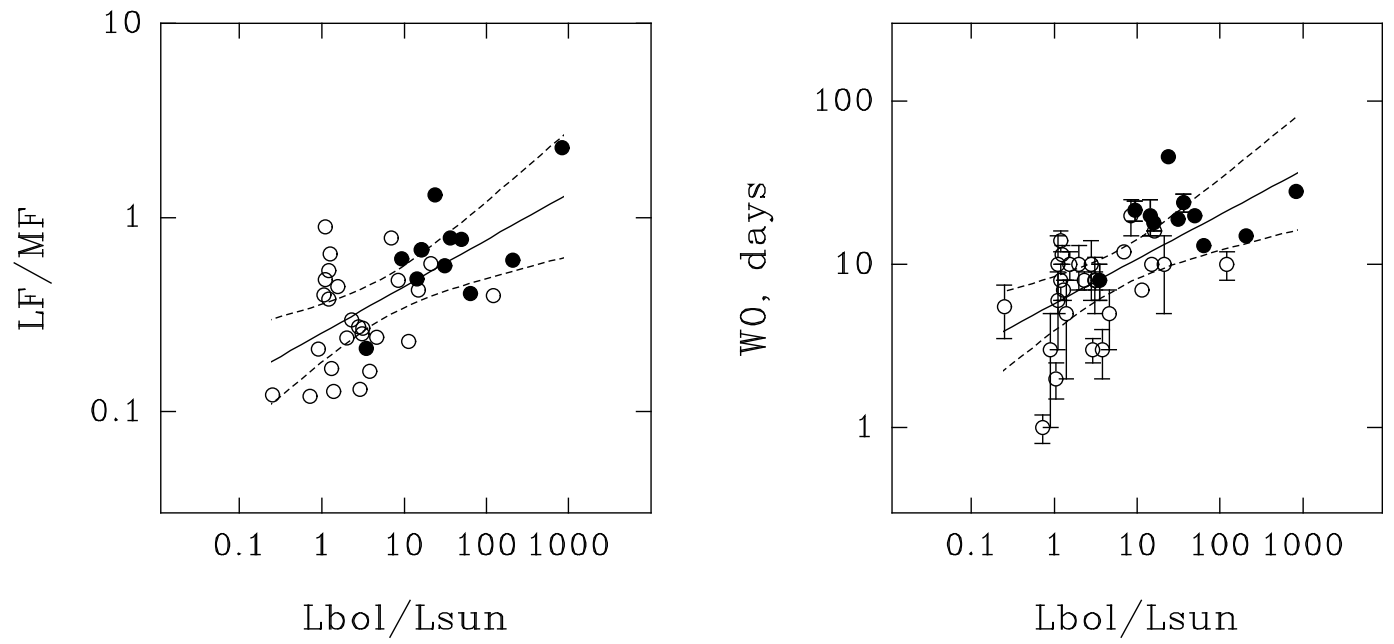

Figure 4: Correlation between the characteristic time of light variability and the total bolometric luminosity of the 'star + disk' system. Open circles: HAeS; filled circles: cTTS

accretion disks by interferometric methods (see, e.g. review [4]). In the near-IR, the spatial resolution of modern interferometers reaches 0.007 arcsec, which corresponds to 0.1 AU at the distance of $140 \mathrm{pc}$. The interferometric observations showed that the inner radius $\mathrm{R}_{\text {in }}$ of accretion disk, where the dust sublimates, is $\sim 0.1 \mathrm{AU}$ in cTTS and $\sim 0.5 \mathrm{AU}$ in HAeS. The values of $R_{\text {in }}$ for the stars under consideration and the references [28,30-34,37,38] are given in columns 4 and 5 in Table 1.

In [31], a relationship between $\mathrm{R}_{\text {in }}$ and the bolometric luminosity of the system "star + disc" was established. Using this relationship, we find dependence of the characteristic time of variability $\mathrm{W} 0$ from $\mathrm{R}_{i n}$. Then, using stellar masses, we find a relationship between $\mathrm{W} 0$ and the Keplerian period at the radius $\mathrm{R}_{i n}$. For some stars, where $\mathrm{R}_{\text {in }}$ is not known, we estimate it from the known luminosity $\mathrm{L}_{t o t}$ and the relationship $\mathrm{R}_{\text {in }}$ vs $\mathrm{L}_{t o t}$ [31]: these estimated $\mathrm{R}_{\text {in }}$ are given in brackets in Table 1.

The empirical relationships in Fig.4, although statistically significant, show rather large scatter of points. Therefore, in the following we analyse only mean values $\left(\mathrm{L}_{t o t}, \mathrm{R}_{i n}, \mathrm{M}, \mathrm{P}_{k e p l}\right.$, W0) calculated for the two groups: cTTS and HAeS. These mean values and the standard deviations of the mean are given in Table 2. It turns out, that the characteristic time of variability $\mathrm{W} 0$ is about $1 / 4$ of the Keplerian period at the inner radius of accretion disk, more precisely - at the radius of dust sublimation (see the last two lines in Table 2.). Apparently, the light variability is related to some processes at the inner radius of the dusty disk.

This is not a trivial result, because the characteristic time of variability is measured from the light curves without any model assumptions, while the Keplerian period is derived from the spectral, photometrical and interferometrical observations and involves the evolutionary tracks and disk models.

\section{Search for stable Keplerian periods}

The obtained results give hopes that Keplerian periods can be detectable in the photomet- 
Table 1: Total bolometric luminosity, inner disk radius, and parameters of photometric variability

\begin{tabular}{|c|c|c|c|c|c|c|}
\hline Object & $\mathrm{L}_{t o t}\left(\mathrm{~L}_{\odot}\right)$ & Reference & $\mathrm{R}_{\text {in }}(\mathrm{AU})$ & Reference & $W 0$ & $L F / M F$ \\
\hline 1 & 2 & 3 & 4 & 5 & 6 & 7 \\
\hline AA Tau & 1.05 & {$[26,27]$} & 0.084 & {$[28]$} & $2.0 \pm 0.5$ & 0.399 \\
\hline AS 205 & 11.4 & {$[29]$} & 0.140 & {$[30]$} & $7.0 \pm 0.2$ & 0.230 \\
\hline BP Tau & 1.25 & {$[26,27]$} & 0.086 & [31] & $11.5 \pm 1.5$ & 0.649 \\
\hline BM And & - & L & 0.249 & [28] & $20.0 \pm 5.0$ & - \\
\hline CI Tau & 1.55 & {$[26,27]$} & 0.097 & [28] & $10.0 \pm 2.0$ & 0.440 \\
\hline DF Tau & 2.93 & {$[26,27,29]$} & 0.090 & [32] & $3.0 \pm 0.5$ & 0.131 \\
\hline DG Tau & 6.90 & {$[26,27,29]$} & 0.142 & [31] & $12.0 \pm 0.3$ & 0.785 \\
\hline DI Cep & 8.50 & [29] & 0.165 & [28] & $20.0 \pm 5.0$ & 0.475 \\
\hline DI Tau & 0.90 & {$[26,27]$} & $(0.10)$ & - & $3.0 \pm 2.0$ & 0.210 \\
\hline DK Tau & 2.80 & {$[26,27]$} & $(0.13)$ & - & $10.0 \pm 4.0$ & 0.273 \\
\hline DL Tau & 1.40 & {$[26,27]$} & $(0.10)$ & - & $5.0 \pm 3.0$ & 0.127 \\
\hline DM Tau & 0.25 & {$[26]$} & $(0.08)$ & - & $5.5 \pm 2.0$ & 0.122 \\
\hline DN Tau & 1.20 & {$[26,27]$} & 0.070 & {$[32]$} & $8.0 \pm 2.0$ & 0.381 \\
\hline DR Tau & 3.80 & {$[26,27,29]$} & 0.103 & {$[32,33]$} & $3.0 \pm 1.0$ & 0.161 \\
\hline DS Tau & 1.10 & {$[26,27]$} & $(0.10)$ & - & $6.0 \pm 3.0$ & 0.479 \\
\hline GG Tau & 2.30 & {$[26,27]$} & $(0.12)$ & - & $8.0 \pm 1.0$ & 0.297 \\
\hline GI Tau & 1.20 & {$[26]$} & $(0.10)$ & - & $14.0 \pm 2.0$ & 0.533 \\
\hline GK Tau & 2.00 & {$[26,27]$} & $(0.12)$ & - & $10.0 \pm 3.0$ & 0.240 \\
\hline GM Aur & 1.10 & {$[26,27]$} & 0.221 & [31] & $10.0 \pm 5.0$ & 0.893 \\
\hline GW Ori & 121 & [29] & $(0.55)$ & - & $10.0 \pm 2.0$ & 0.397 \\
\hline LkCa 15 & 0.72 & {$[26,29]$} & 0.099 & [31] & $1.0 \pm 0.2$ & 0.120 \\
\hline RW AurAB & 3.15 & {$[26,29]$} & 0.130 & {$[28,31]$} & $8.0 \pm 1.0$ & 0.269 \\
\hline RY Tau & 16.3 & {$[26,27,29]$} & 0.300 & [34] & $16.0 \pm 1.0$ & 0.683 \\
\hline SU Aur & 15.0 & {$[26,27]$} & 0.240 & {$[32,33]$} & $10.0 \pm 1.0$ & 0.422 \\
\hline T Tau & 21.1 & {$[26,27,29]$} & $(0.23)$ & - & $10.0 \pm 5.0$ & 0.580 \\
\hline UX TauAB & 1.30 & {$[26]$} & $(0.10)$ & - & $7.0 \pm 1.0$ & 0.167 \\
\hline UY Aur & 3.10 & {$[26]$} & 0.100 & {$[32]$} & $8.0 \pm 3.0$ & 0.251 \\
\hline V1121 Oph & 4.60 & [29] & $(0.15)$ & - & $5.0 \pm 2.0$ & 0.242 \\
\hline BF Ori & 9.40 & [35] & $(0.18)$ & - & $21.5 \pm 3.0$ & 0.612 \\
\hline ВН Сep & 14.4 & {$[36]$} & $(0.20)$ & - & $20.0 \pm 5.0$ & 0.484 \\
\hline CQ Tau & 3.50 & [36] & 0.230 & {$[37]$} & $8.0 \pm 2.0$ & 0.212 \\
\hline KK Oph & 16.0 & {$[29,35]$} & $(0.20)$ & - & $18.0 \pm 2.0$ & 0.683 \\
\hline LkHa 234 & 829 & {$[29,35]$} & $(1.00)$ & - & $28.0 \pm 2.0$ & 2.298 \\
\hline RR Tau & 64.0 & [36] & $(0.45)$ & - & $13.0 \pm 1.0$ & 0.406 \\
\hline SV Cep & 24.0 & [36] & $(0.30)$ & - & $46.0 \pm 2.0$ & 1.113 \\
\hline UX Ori & 31.0 & [36] & 1.090 & [38] & $19.0 \pm 1.0$ & 0.563 \\
\hline VV Ser & 208 & {$[29,35,36]$} & $(0.47)$ & [37] & $15.0 \pm 1.0$ & 0.603 \\
\hline VX Cas & 50.0 & [36] & $(0.40)$ & - & $20.0 \pm 2.0$ & 0.773 \\
\hline WW Vul & 36.3 & [36] & 0.990 & [38] & $24.0 \pm 3.0$ & 0.785 \\
\hline
\end{tabular}


Table 2: Average parameters of cTTS (28 objects) and HAeS (11 objects)

\begin{tabular}{|c|c|c|}
\hline Parameter & cTTS & HAeS \\
\hline $\mathrm{L}_{\text {bol }}\left(\mathrm{L}_{\odot}\right)$ & $8.8 \pm 4.3$ & $116.9 \pm 73.2$ \\
$\mathrm{R}_{\text {in }}(\mathrm{AU})$ & $0.15 \pm 0.02$ & $0.50 \pm 0.11$ \\
$\mathrm{M}\left(\mathrm{M}_{\odot}\right)$ & $0.81 \pm 0.14$ & $3.10 \pm 0.4$ \\
\hline $\mathrm{P}_{\text {kepl }}(\mathrm{d})$ & $25.6 \pm 2.9$ & $86.5 \pm 27.3$ \\
$4 * W 0(\mathrm{~d})$ & $32.6 \pm 3.4$ & $81.4 \pm 10.2$ \\
\hline
\end{tabular}

ric time series. Using the smoothed power spectra, described in Section 3, we composed a list of all the periodic processes with a confidence level above $80 \%$. The periods caused by orbital rotation of low-mass bodies must be stable in time, appearing in different seasons of observations. In order to select such stable periods we inspect phase diagrams for each season individually. In case the periods reveals itself in at least five seasons, we assumed the period as a stable one. As an example, the Fig.5 shows phase diagrams for the star AS 205 for period $\mathrm{P} 1=24.8$ days (the period of axial rotation of the star is 6.78 days). The period P1 can be noticed in the phase diagrams of 1987, 1988, 1989, 1992 and 1993 seasons. Note, that the time interval from 1987 to 1993 covers 88 periods.

Such relatively stable periods were found only in five cTTS: AS 205, CI Tau, DI Cep, GI Tau and GW Ori. Assuming that the periods are caused by orbital motion of bodies (protoplanets) within the protoplanetary disks, and using the stellar masses, we estimated semi-major axes of the orbits. Table 3 lists the stars with relatively stable periods: there are star name, spectral type, effective temperature, stellar luminosity, stellar mass, total number of observations (N1), number of seasons (N2), the period (P) and semi-major axis of the orbit corresponding to the period $\mathrm{P}$.

DI Cep was observed from 1986 to 2003; the period given in Table 3 was detected in data of 1986, 1989, 1991, 1993, 1994 and 1999 seasons, which covers time interval of 40 cycles. GI Tau was observed from 1986 to 1992; the period was detected in 1987, 1988, 1989, 1990 and 1992, which covers 28 cycles. GW Ori was observed from 1987 to 1998 and the period was detected in 1988, 1989, 1992, 1993 and 1998, which covers 45 cycles. Note, that in this paper we do not consider the periods of axial rotation, which are detectable in light curves of many cTTS. The periods of axial rotation are shorter than Keplerian periods and are also quite stable.

Among the HAeS listed in Table 1, we did not find stable periodical variations of light. The long-term cyclic variations with a period of about 650 days are present in SV Cep; this was reported earlier in [12].

\section{Discussion and conclusions}

In this work we analysed long-term UBVR photometry of cTTS and HAeS. We discovered relationship between the characteristic time of variability and the bolometric luminosity of the system "star + disk": the larger the luminosity is, the slower the variability. The relationship is valid over a wide range of masses and luminosities from cTTS to HAeS. Taking into account the known relationship between the inner radius of dusty disk $\mathrm{R}_{\text {in }}$ and the bolometric 

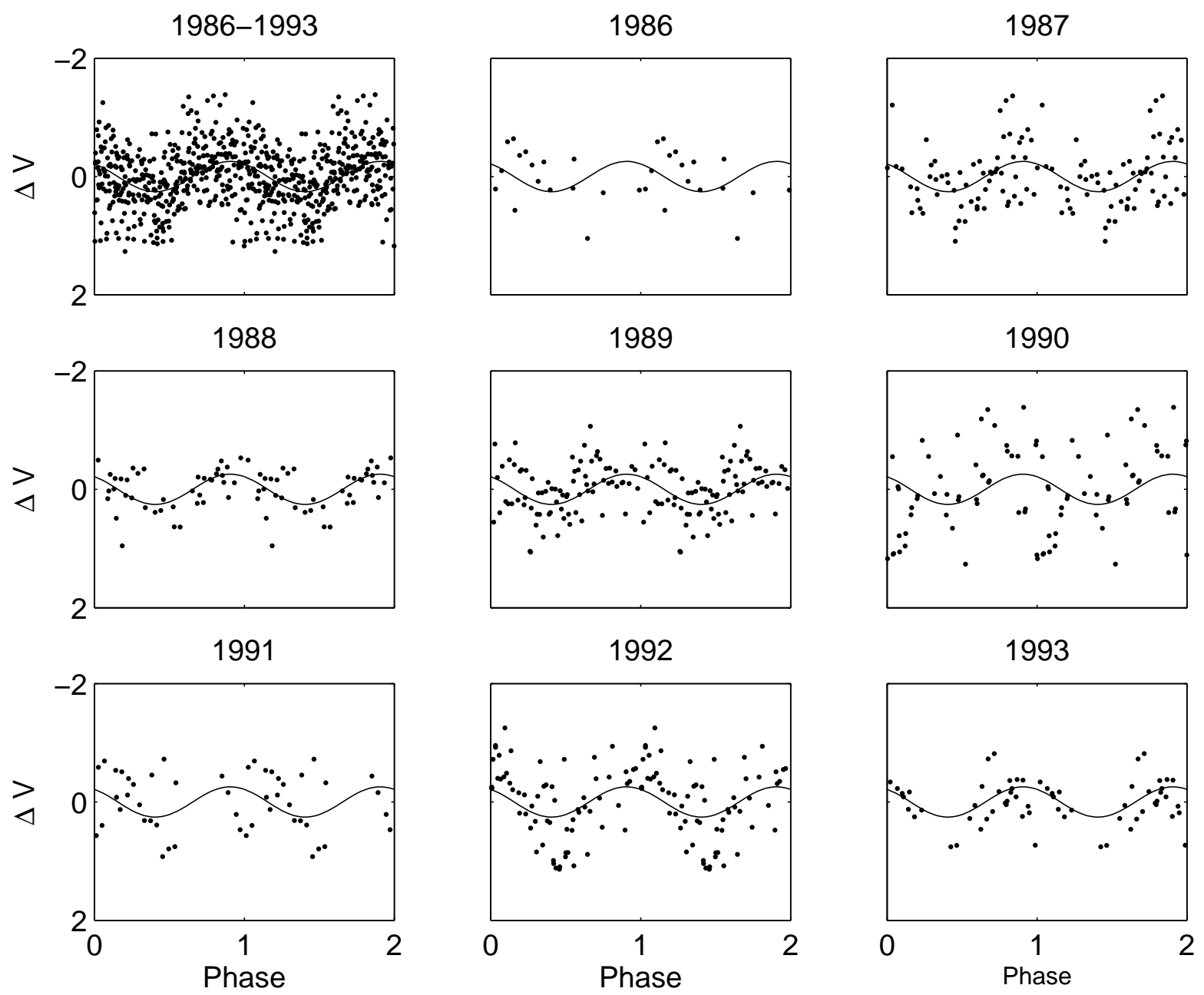

Figure 5: Seasonal light curves of AS205, convolved with period $\mathrm{P}=24.8$ days. Initial epoch $\mathrm{T} 0=2445000$ is the same in each season. The sinusoid fit to the whole set of data is shown in each box 
luminosity, one may suggest that the characteristic time of photometric variability is related to some processes near the inner boundary of the dusty disk. Using the $\mathrm{R}_{i n}$, the mass of the star and the 2nd Kepler's law, we estimated mean Keplerian periods for 28 cTTS and 11 HAeS. It turns out that the observed characteristic time of variability is, on average, equal to $1 / 4$ of the Keplerian period at the radius of dust sublimation.

By means of periodogram analysis of the light curves we found stable periods between 25 and 120 days in five cTTS. This implies existence of stable structures (protoplanets, dust clouds or density waves) within the circumstellar disks, rotating at Keplerian orbits at the distances of 0.1 to $0.5 \mathrm{AU}$ from the star. This is in good agreement with the inner radii of the dusty disks of cTTS known from IR interferometry. Two different mechanisms can be involved in interpretation of these results: 1) circumstellar extinction and 2) accretion. In the first case, motion of a protoplanet causes density waves in the disk and in the disk wind. In the presence of dust in the disk it results in variability of circumstellar extinction, provided a low inclination of light of sight to the disk plane. This mechanism was considered by Tambovtseva and Grinin [43], who argued the ability of dust to survive in the disk winds of cTTS. In the second case, motion of a protoplanet in an eccentric orbit can modulate the rate of accretion and, consequently, the stellar brightness. In this case the light variability can be observed at any inclination of line of sight to the disk plane. The periodic modulation of accretion rate was observed in DQ Tau [44] and V4046 Sgr [45]. Similar scenario was also discussed in [17]. There is also a possibility of a "hybrid" scenario: increase of accretion rate is accompanied by increase of mass-loss, which, in turn, leads to increase in circumstellar extinction. This mechanism was discussed in [46] with reference to V1184 Tau.

One of the most interesting case is the cTTS AS 205A (= V866 Sco). The star shows two distinct periods in light variations, 6.78 and 24.78 days, that persist over several years. The shortest period is due to axial rotation of the star. If the 24.78 days period is due to orbital motion of a gravitating object, there must be also variations in radial velocity of the star. With the mass of the primary $\approx 1 \mathrm{M}_{\odot}$, its orbital velocity is expected to be above $5 \mathrm{~km} \mathrm{~s}^{-1}$ in case the secondary is of stellar mass; from 1 to $5 \mathrm{~km} \mathrm{~s}^{-1}$ in case of a brown dwarf, and below $1 \mathrm{~km} \mathrm{~s}^{-1}$ in case of a planet. The expected amplitude of radial velocity depends on inclination of the orbit to the line of sight, which can be estimated from the observed $v \sin i$, stellar radius and the period of axial rotation (6.78 days). At present, only a few measurements of radial velocity of AS 205 are available, allowing to suspect variability of radial velocity [47]. Note, that if there are spots on stellar surface, variations of radial velocity (within $v \sin i$ ) may appear also due to distortions in photospheric line profiles [48].

Special spectral monitoring of the cTTS, listed in Table 3, is needed to clarify the nature of the periodic brightness variations.

\section{References}

1. P. P. Petrov, Astrophysics 46, 506 (2003).

2. L. B. F. M. Waters, C. Waelkens, Ann. Rev. Astron. Astrophys. 36, 233 (1998).

3. C. A. Grady, The Nature and Evolution of Disks Around Hot Stars, Johnson City, Tennessee, USA (2004). Edited by R. Ignace and K. G. Gayley., ASP Conf. Series 337, 155 (2005).

4. Millan-Gabet, F. Malbet, R. Akeson et al., Protostars \& Planets V, Big Island, Hawaii (2005), 
Edited by B. Reipurth, D. Jewitt, and K. Keil (eds.), Univ. of Arizona Press, p.539 (2007). 5. P. Grinin, N. N. Kiselev, G. P. Chernova et al., Astrophys. Space Sci. 186, 283 (1991). 6. K. N. Grankin, S. Yu. Melnikov, J. Bouvier et al., Astron. Astrophys. 461, 183 (2007). 7. K. N. Grankin, J. Bouvier, W. Herbst, S. Yu. Melnikov, Astron. Astrophys. 479, 827 (2008).

8. V. P. Grinin, L. V. Tambovtseva, N. Ya. Sotnikova, Astron. Lett. 30, 694 (2004).

9. V. P. Tsessevich, V. A. Dragomiretskaya, RW Aur type stars (Kiev: Naukova Dumka, 1973).

10. V. S. Shevchenko, K. Grankin, M. Ibragimov et al., Astrophys. Space Sci. 202, 121 (1993).

11. V. P. Grinin, A. N. Rostopchina, D. N. Shakhovskoi, Astron. Lett. 24, 802 (1998).

12. A. N. Rostopchina, V. P. Grinin, D. N. Shakhovskoi, Astron. Lett. 25, 243 (1999).

13. A. N. Rostopchina, V. P. Grinin, D. N. Shakhovskoi et al., Astron. Rep. 44, 365 (2000)

14. C. Bertout, Astron. Astrophys. 363, 984 (2000).

15. D. N. Shakhovskoi, V. P. Grinin, A. N. Rostopchina, Astrophysics 48, 135 (2005).

16. V. S. Shevchenko, Herbig Ae/Be stars (Tashkent, Izdatel'stvo Fan, 1989, In Russian).

17. W. Herbst, V. S. Shevchenko, Astron. J. 118, 1043 (1999).

18. D. H. Roberts, J. Lehar, J. W. Dreher, Astron. J. 93, 968 (1987).

19. K. Horne, R. A. Wade, P. Szkody, MNRAS 219, 791 (1986).

20. J. H. Horne, S. L. Baliunas, Astrophys. J. 302, 757 (1986).

21. S. L. Marple, Digital spectral analysis with applications (Englewood Cliffs, NJ, Prentice-Hall, Inc., 1987).

22. R. B. Blackman, J. W. Tukey, The measurement of power spectra from the point of view of communication engineering, (New York: Dover Publications, Inc., 1958).

23. V. Yu. Terebizh, Introduction to Statistical Theory of Inverse Problems (Moscow, FIZMATLIT, 2005).

24. G. M. Jenkins, D. G. Watts, Spectral analysis and its applications (Holden-Day Series in Time Series Analysis, London: Holden-Day, 1969).

25. W. Herbst, D. K. Herbst, E. J. Grossman, Astron. J. 108, 1906 (1994).

26. S. J. Kenyon, L. Hartmann, Astrophys. J. Suppl. Ser. 101, 117 (1995).

27. S. Cabrit, S. Edwards, S. E. Strom, K. M. Strom, Astrophys. J. 354, 687 (1990).

28. J. A. Eisner, L. A. Hillenbrand, R. J. White et al., Astrophys. J. 669, 1072 (2007).

29. F. Hamann, S. E. Persson, Astrophys. J. 394, 628 (1992).

30. J. A. Eisner, L. A. Hillenbrand, R. J. White et al., Astrophys. J. 623, 952 (2005).

31. R. L. Akeson, A. F. Boden, J. D. Monnier et al., Astrophys. J. 635, 1173 (2005).

32. J. Muzerolle, N. Calvet, L. Hartmann, P. D'Alessio, Astrophys. J. 597, L149 (2003).

33. R. L. Akeson, C. H. Walker, K. Wood et al., Astrophys. J. 622, 440 (2005).

34. A. A. Schegerer, S. Wolf, Th. Ratzka, Ch. Leinert, Astron. Astrophys. 478, 779 (2008).

35. M. Corcaron, T. P. Ray, Astron. Astrophys. 331, 147 (1998).

36. J. Hernandez, N. Calvet, C. Briceno et al., Astron. J. 127, 1682 (2004).

37. J. A. Eisner, B. F. Lane, L. A. Hillenbrand et al., Astrophys. J. 613, 1049 (2004).

38. J. D. Monnier, R. Millan-Gabet, R. Billmeier et al., Astrophys. J. 624, 832 (2005).

39. P. Hartigan, S. Edwards, L. Ghandour, Astrophys. J. 452, 736 (1995).

40. N. Calvet, J. Muzerolle, C. Briceno et al., Astron. J. 128, 1294 (2004).

41. P. Manoj, H. C. Bhatt, G. Maheswar, S. Muneer, Astrophys. J. 653, 657 (2006).

42. F. D’Antona, I. Mazzitelli, Astrophys. J. Suppl. Ser. 90, 467 (1994).

43. L. V. Tambovtseva, V. P. Grinin, 2008, Astron. Lett. 34, 231 (2008). 
44. G. Basri, Ch. M. Johns-Krull, R. D. Mathieu, Astron. J. 114, 781 (1997).

45. H. C. Stempels, C. F. Gahm, Astron. Astrophys. 421, 1159 (2004).

46. V. P. Grinin, A. A. Arkharov, O. Yu. Barsunova et al., Astron. Lett. 35, 114 (2009).

47. C. H. F. Melo, Astron. Astrophys. 410, 269 (2003).

48. H. C. Stempels, G. F. Gahm, P. P. Petrov, Astron. Astrophys. 461, 253 (2007).

Published in Astronomy Reports, 2010, V.54, P.163 